\title{
Mapping Isobaric Aging onto the Equilibrium Phase Diagram
}

\author{
Niss, Kristine
}

Published in:

Physical Review Letters

DOI:

10.1103/PhysRevLett.119.115703

Publication date:

2017

Document Version

Peer reviewed version

Citation for published version (APA):

Niss, K. (2017). Mapping Isobaric Aging onto the Equilibrium Phase Diagram. Physical Review Letters, 119(11), [115703]. https://doi.org/10.1103/PhysRevLett.119.115703

\section{General rights}

Copyright and moral rights for the publications made accessible in the public portal are retained by the authors and/or other copyright owners and it is a condition of accessing publications that users recognise and abide by the legal requirements associated with these rights.

- Users may download and print one copy of any publication from the public portal for the purpose of private study or research.

- You may not further distribute the material or use it for any profit-making activity or commercial gain.

- You may freely distribute the URL identifying the publication in the public portal.

Take down policy

If you believe that this document breaches copyright please contact rucforsk@kb.dk providing details, and we will remove access to the work immediately and investigate your claim. 


\title{
Mapping isobaric aging onto the equilibrium phase diagram
}

\author{
Kristine Niss \\ Glass \& Time, IMFUFA, Department of Science and Environment, \\ Roskilde University, Postbox 260, DK-4000 Roskilde, Denmark
}

(Dated: August 24, 2017)

\begin{abstract}
The linear volume relaxation and the non-linear volume aging of a glass-forming liquid are measured, directly compared, and used to extract the out-of-equilibrium relaxation time. This opens a window to investigate how the relaxation time depends on temperature, structure, and volume in parts of phase space that are not accessed by the equilibrium liquid. It is found that the temperature dependence of relaxation time is non-Arrhenius even in the isostructural case - challenging the Adam-Gibbs entropy model. Based on the presented data and the idea that aging happens through quasi-equilibrium states, we suggest a mapping of the out-of-equilibrium states during isobaric aging to the equilibrium phase diagram. This mapping implies the existence of isostructural lines in the equilibrium phase diagram. The relaxation time is found to depend on the bath temperature, density, and a just single structural parameter, referred to as an effective temperature.
\end{abstract}

When a liquid is super-cooled well below the melting point the viscosity and characteristic relaxation time increase dramatically. Ultimately, $\tau$ surpasses the experimental timescale, the liquid then falls out of equilibrium and forms a glass. There is consensus that the dynamics close to the glass transition is dominated by "hopping" where energy barriers, $\Delta E$, have to be overcome by thermal fluctuations [1]. This should lead to an Arrhenius temperature dependence of the relaxation time $\tau=\tau_{0} \exp (\Delta E / T)$, where $\tau_{0}$ is a microscopic time. However, for most liquids the temperature dependence is much larger. One of the earliest explanations of the increase in relaxation time was that it could be caused be the decrease in volume upon cooling [2]. However, high pressure measurements have clearly demonstrated that the activation energy depends on both temperature, $T$, and density, $\rho,[3,4]$ leading to an equation of the form $\tau=\tau_{0} \exp (\Delta E(\rho, T) / T)$.

Close to the glass transition, where the relaxation time is in the order of hours, it is possible to perform aging experiments in which the liquid's properties are monitored as it approaches a new equilibrium after a change in temperature [5-8]. The out-of-equilibrium liquid accesses parts of phase space that are not accessed by the equilibrium liquid. It is for example possible to change the volume without a concomitant change of the microscopic structure. Aging experiments can therefore give new information to the puzzle of what governs the relaxation time and could play a role analogous to that played by high pressure measurements [3, 4, 9], ultra stable glasses [10], and confinement $[11,12]$, which have led to important experimental advances in glass science during the last couple of decades.

A long standing phenomenological models for the glasstransition is the Adam-Gibbs model in which the activation energy is governed by the configurational entropy of the liquid [13]. This model has led to the expectation that the relaxation time and viscosity are Arrhenius when the structure of the glass is frozen [14, 15]. However, accessing (and properly defining) the out-of-equilibrium relaxation time is intrinsically challenging, because relaxation time is continuously changing as aging takes place.

In this paper we present a novel strategy for extracting the out-of-equilibrium relaxation time. The method is based on measurements of linear volume relaxation combined with non- linear volume aging. Because the property monitored during aging is volume, the data give direct information about how the relaxation time depends on volume in the out-of-equilibrium liquid. We find that the relaxation time is non-Arrhenius under isostructural conditions - challenging the Adam-Gibbs model. Moreover, we suggest a mapping onto the equilibrium phase diagram implying the nontrivial existence of isostructural lines in the equilibrium phase diagram.

The sample used was polyisobutylene $\mathrm{Mw}=390$ with polydispersity 1.06 acquired from Polymer Standard Service. The technique used for measuring volume relaxation is capacitative dilatometry $[16,17]$. This method takes advantage of the fact that the changes in capacitance are governed by the the density of a dielectric material when studied in a range where no relaxation is present.

The experimental protocol used is to make an "instantaneous" step in temperature and subsequently measure the volume as a function of time. In order for a temperature step to be "instantaneous" compared to the time scale of the relaxation, the latter needs to be at least 2 orders of magnitude larger than the time scale of the temperature change. We find that to have linear response the temperature changes must be as small as $1 \%$ of the absolute temperature which correspond to volume changes in the order of $0.1 \%$. Thus, the requirements for a linear experiment are: precise and fast temperature control combined with high precision measurements of volume changes.

The measurements were performed around the reference temperature $189.7 \mathrm{~K}$ where the dielectric relaxation time is of order one hour, the temperature steps were made on a timescale of a few seconds. The volume change are measured every 2 seconds using an electric signal of $10 \mathrm{kHz}$. (See supplentary material Ref. [18] and Ref. [17] for details).

Fig. 1 depicts the temperature protocol and raw data given in the form of relative volume change $\delta V(t)=\frac{V(t)-V_{\text {ref }}}{V_{\text {ref }}}$ where $V_{\text {ref }}$ is the sample volume at the reference temperature $189.7 \mathrm{~K}$. Up jumps in temperature lead to an increase in volume which takes place in two steps. The first step is a fast glassy expansion analogous to the expansion of crystaline solids. The second step is slow volume aging due to structural relaxation. The equilibrium volume of the reference temperature is recovered after a cycle of an up and a down jump in temperature. 

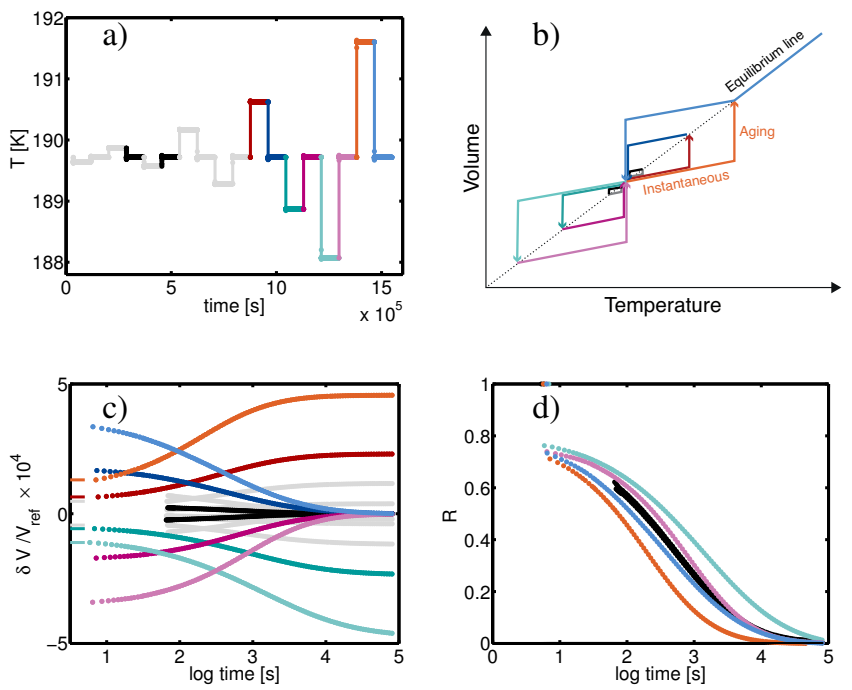

FIG. 1. Protocol and raw data. The colors in this figure are used as the legend for all figures in the paper. a) The measured temperature as a function of time. b) A sketch illustrating the change of volume during the experiment. c) The measured relative changes of volume after each temperature jump as a function of time. d) The normalized relaxation function, $R$, defined in Eq. 1 as a function of time.

The well known asymmetry of the up and down jump [19] is clearly seen even for these moderately sized temperature jumps. For a down jump in temperature the sample moves from a state point of shorter relaxation time to a point of longer relaxation time. As the equilibrium is approached the relaxation thus gets slower leading to autoretardation of the relaxation, which becomes highly stretched. For the up jump the relaxation is conversely autoaccelerated making it less stretched than the linear relaxation.

Only the smallest temperature jumps of $0.2 \mathrm{~K}$ or less lead to symmetric linear response, where the relaxation after up and down jumps has the same shape within the precision of the measurement. This is seen (Fig. $1 \mathrm{~d}$ )) by plotting the normalized relaxation

$$
R(t)=\frac{V(t)-V_{\infty}}{V_{0}-V_{\infty}}
$$

where $V_{0}$ refers to the volume before the temperature jump and $V_{\infty}$ refers to the new equilibrium value.

Equilibrium is never completely achieved because the measurement time is finite. The distance to equilibrium at the end of the experiment is in most cases of order 1\%o, except the largest down jumps in temperature. The estimation of the distance to equilibrium can be crucial for a detailed analysis of the end of the relaxation (see Ref. [20]). The last 3\% of the relaxation is therefore discarded in the analysis.

The aim of this work is to study the behavior of the relaxation time in the out-of-equilibrium liquid in order to determine its dependence of volume, temperature and structure. Within the traditional Tool-Narayanaswamy (TN) formalism [6, 21-23] the relaxation time can be defined as the inverse of the clock rate $\tau(t)=1 / \gamma(t)$. In the TN-formalism the non-linearity is accounted for by introducing a material time, $\xi$ given by the integral

$$
\xi(t)=\int_{0}^{t} \gamma\left(t^{\prime}, T\right) \mathrm{d} t^{\prime}
$$

where $\gamma\left(t^{\prime}, T\right)$ is the so-called "inner clock" rate of the system. The central idea of the TN-formalism is that relaxation function, $R$ is a unique function of $\xi ; R(t)=R(\xi(t))$.

In the traditional use of the TN-formalism one assumes an explicit functions for both the spectral shape of the linear relaxation curve and the temperature dependence of the relaxation time $[6,24]$. However, there are no uniformly agreed-upon functions describing neither of these, in fact the search for such functions is part of the research in glass-science [25-28]. When applying the $\mathrm{TN}$-formalism, the functional choice made can have critical influence on the conclusions drawn as demonstrated by Richert [29]. In this work the linear and non-linear experiments have been performed on the same sample and setup, making it possible to use the $\mathrm{TN}$-formalism to extract the out-of-equilibrium relaxation time without assumptions. Inspired by the formalism developed in Ref. [20, 24] we start by introducing the Kovacs-McKenna rate [30, 31] defined as

$$
\Gamma(t)=-\frac{\mathrm{d} \ln R}{\mathrm{~d} t} .
$$

Fig. 2 a) is a classical Kovacs plot [30] where the KovacsMcKenna rate is shown as a function of the volume. It demonstrates that the Kovacs-McKenna rates can differ even when both volume and bath temperature are the same. The Kovacs-McKenna rate of an exponential relaxation $R(t)=$ $\exp \left(-t / \tau_{\text {exp }}\right)$ is given by one over the relaxation time, $\Gamma_{\exp }=$ $1 / \tau_{\text {exp }}$ i.e. a horizontal straight line in the Kovacs plot. However, non-exponential relaxation as well as non-linearity will lead to deviations from the horizontal line. In Fig. 2 a) it is clearly seen that the linear relaxation (black curves) is very non-exponential, and it is also well known that linear response in glass-formers is stretched. To separate the effect of nonlinearity from that of non-exponentiality we present a rescaled version of the Kovacs plot in Fig. 2 b). Here $\Gamma(t)$ is shown as a function of the normalized relaxation function $R$ from Eq. (1) and it is seen that the linear (black) curves collapse as expected.

From the definition of the clock rate it follows that one can define a dimensionless Kovacs-McKenna rate

$$
\tilde{\Gamma}=-\frac{\mathrm{d} \ln R}{\mathrm{~d} \xi}=-\frac{\mathrm{d} \ln R}{\mathrm{~d} t} \frac{\mathrm{d} t}{\mathrm{~d} \xi}=\frac{\Gamma(t)}{\gamma(t)} .
$$

$\tilde{\Gamma}$ is a function of $R$, because $R$ is a monotone function of $\xi$, meaning that $\tilde{\Gamma}$ falls on a mastercurve when plottet as a function of $R$. The clock rate $\gamma$ is time independent in the case of linear relaxation, thus $\tilde{\Gamma}_{l i n} \propto \Gamma_{l i n}$ and the directly measured linear curve therefore gives the master curve. Hence, the clockrate, can be found from the relation:

$$
\gamma(t)=a \frac{\Gamma(R(t))}{\Gamma_{\text {lin }}\left(R_{\text {lin }}\right)}
$$

evaluating $\Gamma_{\text {lin }}$ in $R_{\text {lin }}=R(t)$. The scaling factor $a$ is set to make the long time limiting value of $\Gamma_{\text {lin }}$ and $\gamma(t)$ match for 

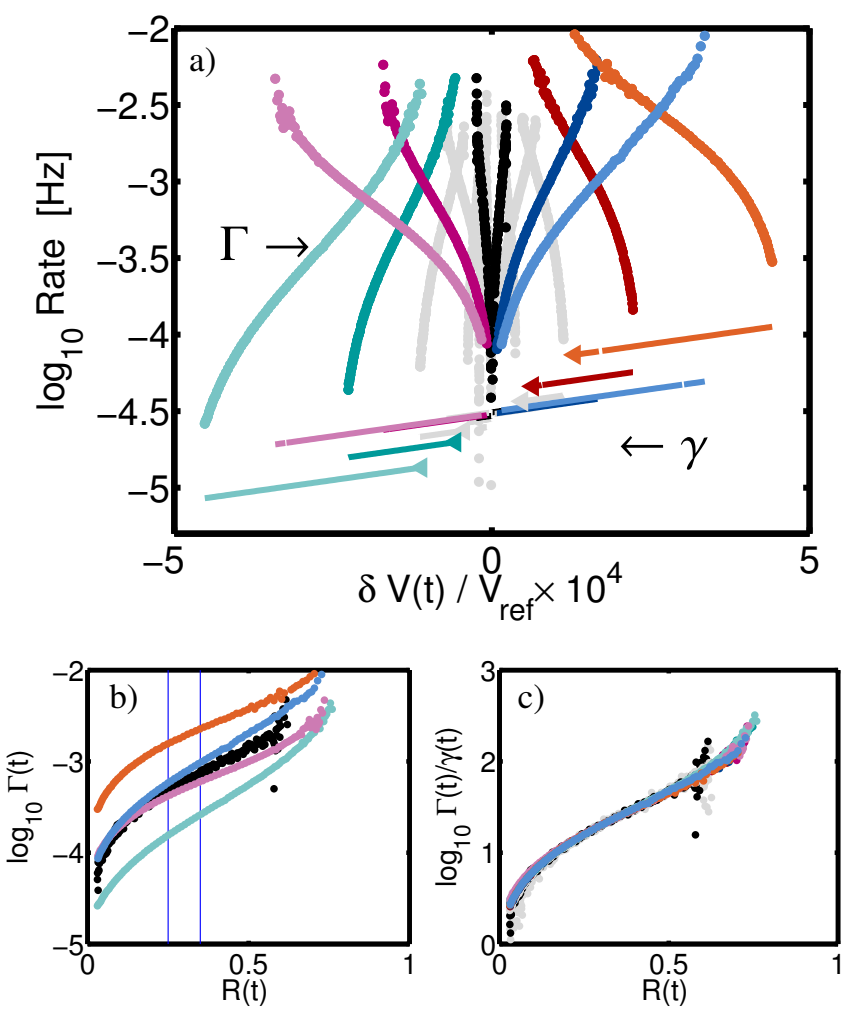

FIG. 2. Kovacs-McKenna rates, $\Gamma$, dimensionless Kovacs-McKenna rates, $\tilde{\Gamma}$, and clock rates, $\gamma$. The colors corrospond to the colors used in Fig.1. Magenta: up-jumps ending at $T=189.7 \mathrm{~K}$, blue: down-jumps ending at $T=189.7 \mathrm{~K}$, red: up-jumps away from $T=189.7 \mathrm{~K}$, green: down-jumps away from $T=189.7 \mathrm{~K}$, black: jumps of $0.2 \mathrm{~K}$, which were found to be linear in Fig. 1 d). a) The Kovacs-McKenna rates (curves in the top of the figure) and clock rates (lines in the bottom of the figure) as a function of volume during aging. Triangles indicate the rates corresponding to the isostructural relaxation times in Fig. 3. b) The Kovacs-McKenna rates as a function of the normalized relaxation function. c) The dimensionless Kovacs-McKenna rates as a function of the normalized relaxation function.

temperature steps ending at $T=189.7 \mathrm{~K}$. Equation (5) is used for finding the clock rate $\gamma(t)$ in the range of $R$ where the data is most precise (illustrated by vertical lines in Fig. 2 (b)). There is noise on the linear curves in Fig. 2 (b) because they are generated from a numerical derivative of a weak signal. A running average is therefore used including both the linear up and down jump. The extracted $\log (\gamma(t))$ values are found to be a linear function of volume with the same slope for all temperature jumps, extending the findings of Ref. [32], but at odds with the suggestion in Ref. [33]. The linear dependence of $\log (\gamma(t))$ on volume is inserted in Eq. (4) to find $\tilde{\Gamma}$. It makes all the relaxation curves collapse in the dimensionless Kovacs plot as shown in Fig. 2 (c). This demonstrates that the data are consistent with the TN-formalism and a linear dependence of $\log (\gamma(t))$ on volume. The clock rate extracted from the linearization is plotted along with the Kovacs-McKenna rate in Fig. 2 (a).

A close study of Fig. 2 (a) reveals that the rates of all the jumps with the same final temperature fall on one line,

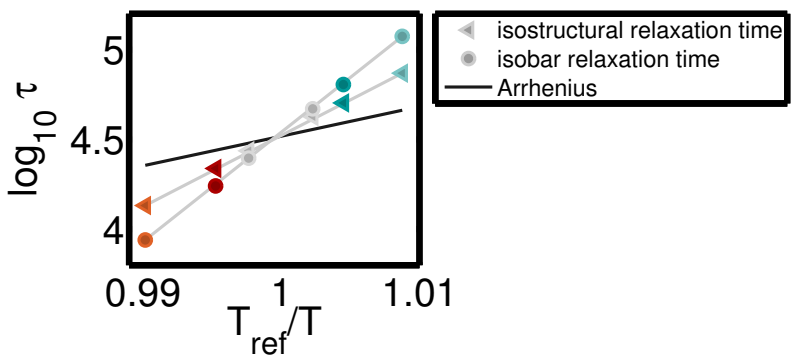

FIG. 3. Temperature dependence of relaxation time around $T_{\text {ref }}=189.7 \mathrm{~K}$. The isostructural relaxation time is the relaxation time found after the rapid isostructural volume change following a temperature jump away from $T_{\text {ref }}$. The corresponding rates are indicated by triangles in Fig. 2 a) and they are found by evaluating $\gamma(T, V)$ at the bath temperature and the volumes corresponding to the short time plateaus in Fig. $1 \mathrm{c}$ ). The Arrhenius line is found by assuming a microscopic time $\tau_{0}$ of a picosecond.

meaning that there is no memory effect on the clock rate. Thus there is no explicit dependence on time and we can write $\log (\gamma(t))=\log (\gamma(V(t), T))$ during isobaric aging. This indicates that if there are dynamical heterogenities and/or local fluctuations in the dynamics then these all age in the same way as "slaves" of the global clock rate $\gamma(t)$ in agreement with earlier findings [29, 34]. Now we can consistently define the out-of-equilibrium relaxation time as one over the rate $\tau(t)=1 / \gamma(t)$ and analyze how $\tau(t)$ depends on the state of the liquid.

When the temperature is changed there is an initial volume change, on time scales much shorter than the structural relaxation. The fast volume change corresponds to the glassy response, and accounts for more than $20 \%$ of the total density change (Fig. 1 d)). It has a contribution from anharmoncity analogous to expansion of chrystaline solids and possibly also a contribution from a $\beta$-relaxation process. With the current setup we cannot separate these two contributions, however the $\beta$-relaxation in the dielectric spectrum accounts for few percent of the total relaxation. Moreover, it is generally believed that the $\beta$-relaxation is local in nature. Therefore we assume that the volume change which is faster than the structural relaxation is isostructural. Along with the temperature change and the isostructural volume change there is also a fast - isostructural - change in the relaxation time $\tau(t)$. By considering the relaxation time right after a series of temperature jumps starting from $189.7 \mathrm{~K}$, we get access to the isostructural temperature dependence of the relaxation time. The result is shown in Fig. (3) along with the equilibrium (isobaric) temperature dependence and the Arrhenius dependence expected in the case of a constant activation energy. Namely $\tau=\tau_{0} \exp \left[(\Delta E) /\left(k_{B} T\right)\right]$ setting $\tau_{0}$ to a physically relevant value of one picosecond. In Fig. (3) the isostructural points clearly deviate from this Arrhenius behavior, demonstrating that there is a change in the activation energy even under isostructural conditions. We assume, as mentioned above, that the glassy response is isostructural and our finding challenges the Adam-Gibbs model under this assumption, because the model has configurational entropy as the governing parameter. 


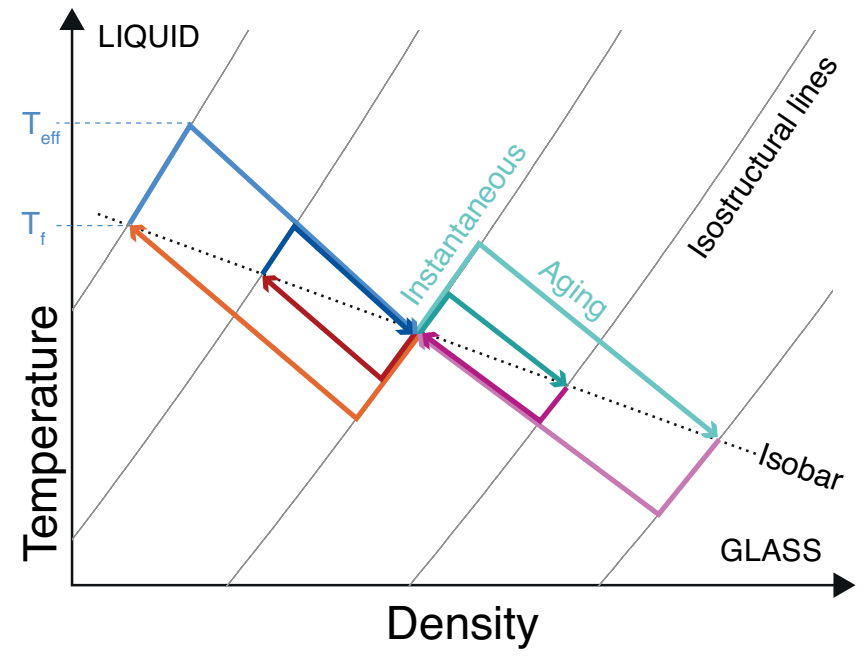

FIG. 4. Illustration of the suggested paths in $T$ - $\rho$-phase diagram. The colors corrospond to the colors used in Fig.1. The temperature scale represents actual temperature in equilibrium whereas it is the effective temperature when the sample is out of equilibrium. $T_{\text {eff }}$ and $T_{f}$ marked on the y-axis correspond to the state of the liquid right after the largest down jump in temperature (blue curve) and indicate the position of the effective temperature and the classical fictive temperature.

We now move on to a global interpretation of the relaxation time's dependence on the state of the liquid. The fact that the relaxation time is the same when density and bath temperature is the same during isobaric aging with different jump sizes suggests that the out-of-equilibrium liquid moves through the same structural states for different jumps with the same final temperature. This can be rationalized via the notion that the transient states during aging are quasi-equilibrium states $[35,36]$, an idea that lies behind the generalized fluctuation dissipation theorem (FDT) [37, 38] and the definition of an effective temperature $[39,40]$.

Here we build on the idea that aging happens by visiting quasi-equilibrium states and suggest a mapping of isobaric aging onto the equilibrium phase diagram. The starting and end points are by definition on the isobar. The jump in temperature leads to a fast isostructural volume change, moving the sample to a state which has the same structure yet a different volume than the initial point. The idea of the mapping implies that this new point corresponds to an equilibrium point which could be the start of another jump (though it would be performed at a different pressure), which would take the liquid to yet another isostructural point. Consequently fast isostructural volume changes after a temperature jump, combined with the conjecture of a mapping from out-of-equilibrium to equilibrium state points, imply the existence of isostructural lines in the equilibrium phase diagram.

Guided by the above arguments we follow the ideas of Gnan et al. [36] who based on the isomorph theory suggest a phase diagram with isostructural lines (so-called isomorphs). In order to describe the aging path in the equlibrium phase diagram (Fig. 4 ) an effective temperature is defined by the equilibrium point that has the same structure and density as the out-ofequilibrium liquid.

When temperature is decreased density increases in two steps - first fast while staying on the isostructural line and then slowly by structural aging. From the raw data we know that the density change during the isostructural step is of the order $20-30 \%$ of the total density change. Our conjecture is that the activation energy depends uniquely on density and the effective temperature. The effective temperature from Gnan is defined coming from a generalized FDT $[37,40]$, and is from a different tradition of aging studies than this work. However, if the notion of a mapping from out-of-equilibrium to equilibrium is to be meaningful, the two types of "structural" temperature must be identical.

Note that the effective temperature defined here is different from the classical "fictive temperature" which is often introduced when treating (isobaric) aging experiment [6, 21, 24]. In the classic definition the fictive temperature is the temperature on the experimental (atmospheric) isobar where the equilibrium liquid structure corresponds to the structure of the out-of-equilbrium liquid. Right after an instantaneous temperature jump the fictive temperature is therefore equal to the temperature before the jump. The difference between the two temperatures is illustrated in Fig. (4). The classical fictive temperature definition de facto ignores that the equilibrium phase diagram has two dimensions and cannot be generalized to describe aging after a pressure jump.

In conclusion we find that the there is no memory effect on the relaxation time of the out-of-equilibrium liquid. We suggest a mapping of the isobaric aging path onto the equilibrium phase diagram by defining an effective temperature. The activation energy then depends on the effective temperature and the density. Specifically, it is found that the activation energy can change even when structure is constant, a finding that challenges the Adam-Gibbs model. Moreover, we argue that the mapping implies the existence of isostructural lines in the equilibrium phase diagram. This suggests that understanding isobaric aging of simple liquids in the framework of isomorph theory [41] might be a promising route.

\section{ACKNOWLEDGEMENTS}

Jeppe Dyre, Tina Hecksher and Ludovic Berthier are thanked for useful discussions. This work has been funded by the Danish Council for Independent Research (Sapere Aude: Starting Grant), the Danish National Research Foundation (DNRF) and L'Oreal Denmark for Women in Science. 
[3] M. L. Ferrer, C. Lawrence, B. G. Demirjian, D. Kivelson, C. Alba-Simionesco, and G. Tarjus, J. Chem. Phys. 109, 8010 (1998).

[4] C. M. Roland, S. Hensel-Bielowka, M. Paluch, and R. Casalini, Rep. Prog. Phys. 68, 1405 (2005).

[5] L. C. E. Struik, Physical Aging in Amorphous Polymers and Other Materials (Elsevier, Amsterdam, 1978).

[6] G. W. Schere, Relaxation in Glass and Composites, edited by Wiley (New York, 1986).

[7] R. Casalini and C. M. Roland, Phys. Rev. Lett. 102, 035701 (2009).

[8] S. L. Simon and G. B. McKenna, in Polymer Glasses, edited by C. B. Roth (Taylor \& Francis Group, 2016).

[9] K. Koperwas, A. Grzybowski, K. Grzybowska, Z. Wojnarowska, A. P. Sokolov, and M. Paluch, Phys. Rev. Lett. 111, 125701 (2013).

[10] S. F. Swallen, K. L. Kearns, M. K. Mapes, Y. S. Kim, R. J. McMahon, M. D. Ediger, T. Wu, L. Yu, and S. Satija, Science 315, 353 (2007).

[11] J.-Y. Park and G. B. McKenna, Phys. Rev. B 61, 6667 (2000).

[12] K. Adrjanowicz, K. Kaminski, M. Dulski, P. Wlodarczyk, G. Bartkowiak, L. Popenda, S. Jurga, J. Kujawski, J. Kruk, M. K. Bernard, and M. Paluch, The Journal of Chemical Physics 139, 111103 (2013), http://dx.doi.org/10.1063/1.4820492.

[13] G. Adam and J. H. Gibbs, J. Chem. Phys. , 139 (1965).

[14] I. M. Hodge, Macromol. 20, 2897 (1987).

[15] J. C. Mauro, D. C. Allan, and M. Potuzak, Phys. Rev. B 80, 094204 (2009).

[16] C. Bauer, R. Böhmer, S. Moreno-Flores, R. Richert, H. Sillescu, and D. Neher, Phys. Rev. E 61, 1755 (2000).

[17] K. Niss, D. Gundermann, T. Christensen, and J. C. Dyre, Phys. Rev. E 85, 041501 (2012).

[18] See Supplemental Material in the appendix.

[19] D. Cangialosi, V. M. Boucher, A. Alegria, and J. Colmenero, Soft Matter 9, 8619 (2013).
[20] T. Hecksher, N. B. Olsen, K. Niss, and J. C. Dyre, J. Chem. Phys. 133, 174514 (2010).

[21] A. Q. Tool, J. Am Ceram. Soc. 29, 240 (1946).

[22] O. S. Narayanaswamy, J. Am. Ceram. Soc. 54, 491 (1971).

[23] J. C. Dyre, J. Chem. Phys. 143, 114507 (2015).

[24] T. Hecksher, Relaxation in supercooled liquids. Linear and Nonlinear, Mechanical and Dielectric Studies of Molecular Liquids, Ph.D. thesis, Roskilde University (2010).

[25] T. Hecksher, A. I. Nielsen, N. B. Olsen, and J. C. Dyre, Nature Physics 4, 737 (2008).

[26] Q. Zheng and J. C. Mauro, J.Am. Ceram. Soc. 100, 6 (2017).

[27] T. Blochowicz, C. Tschirwitz, S. Benkhof, and E. A. Rössler, J. Chem. Phys. 118, 7544 (2003).

[28] N. Sağlanmak, A. I. Nielsen, N. B. Olsen, J. C. Dyre, and K. Niss, J. Chem. Phys 132, 024503 (2010).

[29] S. K. S. Mazinani and R. Richert, J. Chem. Phys. 136, 174515 (2012).

[30] A. J. Kovacs, Fortschr. Hochpolym.-Forsch 3, 394 (1963).

[31] G. McKenna, J. Res. Natl. Inst. Stand. Technol. 99, 169 (1994).

[32] T. Hecksher, N. B. Olsen, and J. C. Dyre, J. Chem. Phys. 142, 241103 (2015).

[33] P. Bernazzani and S. L. Simon, J. Non-Cryst Solids 307-310, 470 (2002).

[34] K. Vollmayr-Lee, C. H. Gorman, and H. E. Castillo, J. Chem. Phys. 144, 234510 (2016).

[35] S. Franz and G. Parisi, J. of Stat. Mech 2013, P02003 (2013).

[36] N. Gnan, C. Maggi, T. B. Schrøder, and J. C. Dyre, Phys. Rev. Lett. 104, 125902 (2010).

[37] L. F. Cugliandolo and J. Kurchan, Phys. Rev. Lett. 71, 173 (1993).

[38] S. Franz, M. Mézard, G. Parisi, and L. Peliti, Phys. Rev. Lett. 81, 1758 (1998).

[39] L. F. Cugliandolo, J. Kurchan, and L. Peliti, Phys. Rev. E 55, 3898 (1997).

[40] J. Kurchan, Nature 433, 222 (2005).

[41] J. C. Dyre, J. Phys. Chem. B 118, 10007 (2014). 